\title{
A Correspondence Analysis of Nine Japanese Historical English-as-a-Foreign-Language Textbooks
}

\author{
Ryohei Honda, Kiyomi Watanabe \\ Fukuyama Heisei University \\ Fukuyama, Japan \\ Toshiaki Ozasa \\ Hiroshima University (Professor Emeritus) \\ Higashi-Hiroshima, Japan
}

\begin{abstract}
The present paper aims to quantitatively describe and explain the features of nine Japanese historical EFL textbooks and their current counterpart, by using a correspondence analysis (CA), focusing on their similarities / differences. The following are the obtained results. First, the CA results proved capable of differentiating the features of the nine historical textbooks and their current counterpart quantitatively, showing their similarities and differences. In particular, the CA map comprised of the two major dimensions indicated that the ten textbooks acan be divided into four groups and that it was only Dimension 1 or the axis of 'difficult vs. easy texts' that differentiated the two major groups. Second, when compared with their current junior-high counterpart, Sunshine, all the textbooks, except for Seisoku, StandardP and Globe, proved to be similar to each other in terms of the 'drillcentered vs. natural' category (Dim 2). This suggests that the six historical textbooks are similar to their current counterpart, Sunshine and that the other three textbooks are different from their current counterpart in terms of the drill-centered vs. natural' texts category. Third, the explaining categories (dimensions, axes) proved to be the 'difficult vs. easy' texts axis (Dim 1), the 'drill-centered vs. natural' structure axis (Dim 2), 'multi-viewpoints vs. single viewpoint' discourse axis (Dim 3), 'redundant vs. concise' texts axis (Dim 4) and 'children's vs. adults' viewpoint' axis (Dim 5).' Finally, it was concluded that the results of the present study suggest that CA is a useful tool for describing, interpreting and diagnosing the features of Japanese EFL textbooks.
\end{abstract}

Keywords: Correspondence analysis; Japanese EFL textbooks; Corpora. 


\section{Introduction}

It has to be stated first of all that the qualitative and quantitative analysis of Japanese historical English-as-a-foreign-language (henceforth EFL) textbooks is a narrow, limited area with very few researchers participating in the development and accumulation of the academic expertise in this field. This is probably because the research itself necessarily takes a time-consuming and energy-consuming process, which has to start with collecting relevant data scattered around and digitizing the collected historical data for quantitative analysis, transforming their graphic texts into digital corpora.

Presumably, Ozasa and Nakamura (2001) was the first academic contribution to the qualitative analysis of Japanese historical EFL textbooks in Japan, which, after presenting a bibliographical introduction of the eight Japanese historical EFL textbooks, depicted the contents of the eight textbooks based on a close reading of the texts. Ozasa and Erikawa (Eds.) (2004), the second contribution to the area, was a more comprehensive diachronic analysis of Japanese historical textbooks, this time, both qualitative and quantitative. In this study, sixteen historical textbooks were described and quantitatively analyzed, covering most of the representative ones, and nine textbooks, including H. E. Palmer's The Standard English Readers, were quantitatively analyzed in terms of nine lexical, grammatical and readability indices. It is to be noted in this respect that the readability measuring indices used in this study and the following ones were widely-known, universal ones, i.e., Flesch Reading Ease and Flesch-Kincaid Grade Level.

In 2007, Nakamura and Ozasa analyzed two corpus databases, (1) fourteen EFL textbook series used in Japan across a span of 135 years and (2) five Asian EFL textbooks, in terms of gender awareness, while Weir and Ozasa (2007) measured the naturalness of the discourse of three Japanese historical EFL textbooks as denoted by their appropriateness to the parts-of-speech profile represented by the American English Brown corpus.

Further, in this context, Weir and Ozasa (Eds.) (2007) and Weir and Ozasa (Eds.) (2008) reported various kinds of synchronic and diachronic textbook studies using the techniques of corpus analysis, covering the areas of grammar, vocabulary, lexical collocation, semantics, readability, language acquisition, etc., among others. Specifically, Ozasa, Umamoto, Matuoka and Motooka (2008) reported on the diachronic comparison of the first year books of twenty-three historical EFL textbooks and one current one, focusing on overall tokens, types and new types, and Matsuoka, Umamoto, Ozasa and Motooka (2008) reported on the diachronic comparison of the same set of EFL textbooks, focusing on the frequency of the past and the present forms of be-verbs and general verbs, while Hosaka and his team reported a diachronic comparison of seven Japanese historical EFL textbooks focusing on the occurrences of toinfinitive forms (Hosaka, Abe, Uenishi \& Ozasa, 2008) and of verbal forms (Hosaka, Erikawa, Miura \& Ranjan, 2008).

Weir, specialist in computer science and computer programming, put his high-tech expertise into practical use in the quantitative analysis of EFL textbook corpora. Weir and Ozasa (2008) computed and compared the frequency of individual words and multiword sequences (n-grams) across three Japanese historical EFL textbook corpora. Weir and Ozasa (2009) performed a 
test of the assumption that three sets of Japanese historical EFL textbooks exhibit priority of grammatical over vocabulary consideration. Weir and Ozasa (2010) reported on the application of computer-based text analysis tools, using three Japanese EFL textbook corpora, in terms of n-gram frequency, hapax legomena and Dolch word.

In 2016, a new attempt was made with an innovative approach in Kawamura, Umamoto and Ozasa and Kawamura, Umamoto, Matsuoka and Ozasa (2017), in which, using the same textbook corpora analyzed in Ozasa and Erikawa (Eds.) (2004), new quantitative analyses were carried out by using a newly developed readability measuring tools, Ozasa-Fukui Year Level, Ver. 3.4.2nhnc1-5 (Ozasa, Fukui \& Watanabe, 2015) and Ver. 3.5nhnc1-6 (Ozasa, Watanabe \& Fukui, 2016).

These studies were further followed by three related studies, Sakamoto, Watanabe and Ozasa (2017), Watanabe, Asai and Ozasa (2017) and Uenishi, Watanabe and Ozasa (2017). In Sakamoto et al, a correspondence analysis was carried out using five Japanese historical EFL textbooks and their current counterpart. In Watanabe et al, the same type of corpus analysis was performed using five different Japanese historical EFL textbooks and two of their current counterparts. Again in Uenishi et al, the same type of quantitative analysis was performed, this time, using six Japanese current EFL textbook corpora, in order to quantitatively examine their similarities / differences.

The present study, motivated by the same awareness and goals as in the preceding ones, aims to quantitatively analyze the same textbook corpora as in Ozasa and Erikawa (Eds.) (2004), from a different perspective, using a different, new corpus analysis technique, i.e., correspondence analysis (Henceforth CA).

\section{Aim}

The present paper aims to quantitatively describe and explain the features of nine Japanese historical EFL textbooks and their current counterpart, by using a CA, focusing on their similarities / differences. The following are the research questions of the present paper:

(1) How similar / different in contents are the nine Japanese historical EFL textbooks to / from each other?

(2) How similar / different in contents are the nine Japanese historical EFL textbooks to / from their current counterpart?

(3) What kinds of dimensions explain the similarities / differences among the ten textbooks?

The textbooks used in the present CA were nine historical EFL textbooks used during the period from 1861 to 1952, and a currently used EFL textbook, which was included in the present analysis for comparative purposes (Ozasa \& Erikawa, (Eds.), 2004). They were the following.

1) New English Drill Books 1 (Kenjiro Kumamoto, 1907, Kaiseikan) (Henceforth

Drill.) 
2) New Jack and Betty: English Step by Step 1 (K. Hagiwara, M. Inamura \& K. Takezawa, 1952, Kairyudo) (Henceforth Jack \& Betty.)

3) The Standard English Readers 1 (Tsuneta Takehara, 1932, Taishukan) (Henceforth StandardT.)

4) Sanders' Union Readers 1 (Charles Walton Sanders, 1861, Ivison, Blakeman, Taylor \& Co.) (Henceforth Union.)

5) New National Readers 1 (C. J. Barnes, 1883, A. S. Barnes \& Co.) (Henceforth National.)

6) English Readers: The High School Series 1 (Education Department, Japan (W. Dening, 1887, Education Department Publishing, Japan ) (Henceforth Dening.)

7) Seisoku Education Department English Reader 1 (Education Department, Japan, 1889, Education Department Publishing, Japan) (Henceforth Seisoku.)

8) The Globe Readers 1 (Yoshisaburo Okakura, 1907, Dainippon Tosho) (Henceforth Globe.)

9) The Standard English Readers 1 (H. E. Palmer, 1926, Institute for Research in English Teaching, Japan) (Henceforth StandardP.)

10) Sunshine English Course I (Masao Niizato, et al, 2012, Kairyudo) (Henceforth Sunshine.)

Through this analysis, we hope to quantitatively clarify the similarities / differences of the nine Japanese historical textbooks and their relationships with their current counterpart.

\section{Method}

The present study employed a one-way CA model with ten nominal variants, in order to explore the relationships among the ten Japanese EFL textbooks, i.e., Drill, Jack \& Betty, StandardT, Union, National, Dening, Seisoku, Globe, StandardP and Sunshine.

First, a cross tabulation table of the above-mentioned ten textbooks was prepared as a basic datum for the present CA, using the ten textbook corpora. This is practically a cross-textbook word-frequency table, which was made using AntConc, a vocabulary processing tool. In this frequency table the most frequent 100 words (content words and function words) were picked up from the frequency list and their frequency values were placed for each of the ten textbooks. (Since the ten kinds of 100 word lists were incorporated into one cross-frequency word table, the number of words of the cross tabulation table was more than 100.)

Then, using the digital datum of the cross tabulation table, CAs were carried out by using Fukui's College Analysis, a statistics computer program developed for social studies by Masayasu Fukui, professor of applied mathematics at Fukuyama Heisei University, Japan. In evaluating and interpreting the results of the analysis, some of the ideas and techniques developed in Tono (2000) were used for reference where they were deemed relevant and appropriate to the topics dealt with in the present paper.

\section{Results and Discussion}

Table 1 shows the basic statistics of the present CA, i.e., the eigenvalues, coefficient correlations, contribution rates and cumulative contribution rates of 
the analysis. As the contribution rate indicates in Table 1, the relationship among the ten nominal variants (textbooks) were explained $31.6 \%$ by the first dimension, $16.8 \%$ by the second dimension, $13.8 \%$ by the third dimension, $11.7 \%$ by the fourth dimension and $10.2 \%$ by the fifth dimension, the cumulative contribution rate being $84.1 \%$ on the fifth dimension. This means that the five dimensions should be included in the analysis and interpretation of the present corpora.

Table 1. Basic CA Data, Ten Textbooks)

\begin{tabular}{|l|r|r|r|r|r|r|r|r|r|}
\hline & \multicolumn{1}{|c|}{$\operatorname{Dim} 1$} & $\operatorname{Dim} 2$ & $\operatorname{Dim} 3$ & \multicolumn{1}{l}{ Dim 4 } & $\operatorname{Dim} 5$ & $\operatorname{Dim} 6$ & $\operatorname{Dim} 7$ & $\operatorname{Dim} 8$ & $\operatorname{Dim} 9$ \\
\hline Eigenvalue & 0.102 & 0.054 & 0.045 & 0.038 & 0.033 & 0.021 & 0.014 & 0.009 & 0.008 \\
\hline Correlation & 0.319 & 0.233 & 0.212 & 0.195 & 0.181 & 0.144 & 0.116 & 0.097 & 0.089 \\
\hline Contribution rate & 0.316 & 0.168 & 0.138 & 0.117 & 0.102 & 0.064 & 0.042 & 0.029 & 0.024 \\
\hline $\begin{array}{l}\text { Cumulative } \\
\text { contribution rate }\end{array}$ & 0.316 & 0.483 & 0.622 & 0.739 & 0.841 & 0.904 & 0.946 & 0.976 & 1 \\
\hline
\end{tabular}

Table 2 shows the values of the ten textbooks on the five dimensions and Figure 1 visualizes the CA results of the ten textbooks on Dim 1, as a description of the relationships among the ten variants. As it is clear in Table 2 and Figure 1, on the first dimension, the value is the highest for Dening (1.864), the second highest for Union (1.142), and the lowest for Sunshine (-1.564), the second lowest for Jack \& Betty (-1.088), the third lowest for Drill (-0.914), while National, StandardT, Globe, StandardP and Seisoku coming in between them. In decreasing order, the ten textbooks were: Dening $>$ Union $>$ National $>$ StandardT $>$ Globe $>$ StandardP $>$ Seisoku $>$ Drill $>$ Jack E Betty $>$ Sunshine.

Table 2. Values of Ten Textbooks on Five Dimensions

\begin{tabular}{|l|r|r|r|r|r|}
\hline & \multicolumn{1}{|c|}{ Dim 1 } & \multicolumn{1}{|c|}{ Dim 2 } & Dim 3 & \multicolumn{1}{c|}{ Dim 4 } & Dim 5 \\
\hline StandardP & -0.252 & -0.862 & -0.544 & 0.576 & -1.027 \\
\hline StandardT & 0.591 & -0.224 & 0.103 & 0.056 & -0.084 \\
\hline Jack\&Betty & -1.088 & -0.021 & -0.235 & 0.44 & 0.201 \\
\hline Union & 1.142 & 0.403 & 0.869 & -0.176 & -0.696 \\
\hline Seisoku & -0.316 & 2.129 & -1.413 & -0.963 & 0.696 \\
\hline Drill & -0.914 & -0.154 & -0.398 & -0.242 & -2.044 \\
\hline National & 0.708 & -0.119 & 1.546 & -1.788 & 0.094 \\
\hline Globe & 0.273 & -1.953 & -1.181 & -0.608 & 1.441 \\
\hline Dening & 1.864 & 0.592 & -0.296 & 2.039 & 0.241 \\
\hline Sunshine & -1.564 & 0.204 & 1.628 & 1.019 & 1.159 \\
\hline
\end{tabular}

$\operatorname{Dim} 1$

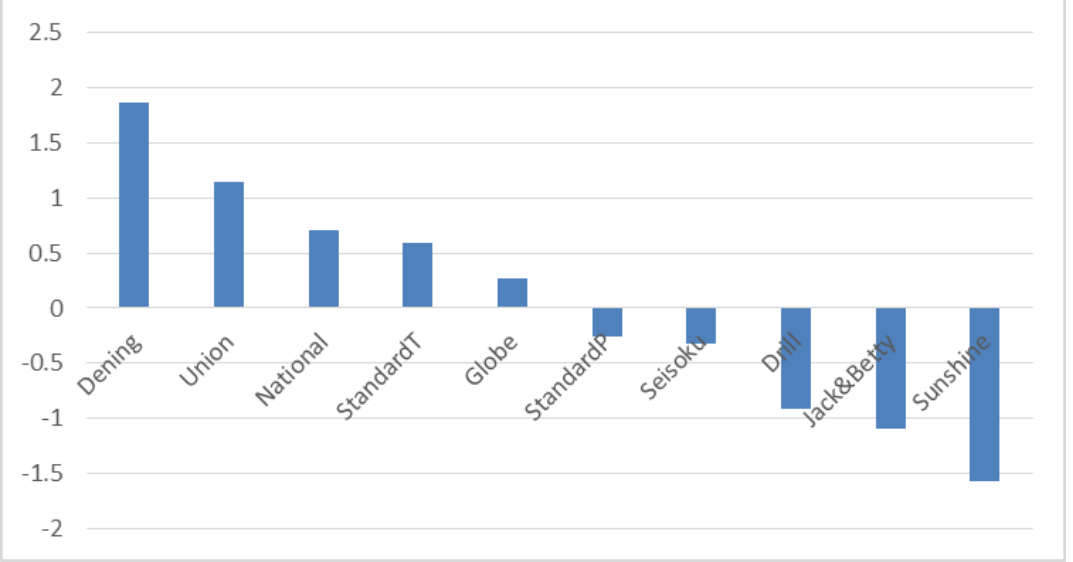

Figure 1. Values of Ten Textbooks on Dim 1 
The differences / similarities among the ten textbooks on the first dimension could best be explained by the category of 'difficult vs. easy texts'; in Dening, Union and National, grammatical and lexical items were not strictly controlled, allowing more freedom for natural communication and communicative activity while in Sunshine and Jack \& Betty, the texts were written using a small, limited number of basic grammatical and lexical items in every lesson.

For example, when the following two pieces of the first lessons of the two contrasting textbooks, Dening and Sunshine, are compared, it is obvious that in Dening the text sounds more natural and therefore more challenging for fresh EFL learners and their readability is contrastively high while Sunshine uses easy and basic patterns repeatedly. For this reason, the first dimension was termed 'difficult vs. easy texts'. The contribution rate of the first dimension was 31.6\%, covering approximately one-third of the whole contribution.

(Dening) Once upon a time a clam, who had opened his shell wide, was basking in the sunshine on the sea shore. A snipe happened to see what was going on. (Lesson 1)

(Sunshine) Hi, I am Saki. Oh, you are Saki. I'm Tom. (Program 2. Program 1 is on Classroom English.)

It is to be noted at this stage that this CA readability judgment is not a direct, measurement-based estimation like readability measurement but an indirect one based on a highly advanced correlation-based mathematical processing of cross-frequency tabulation of the top one-hundred words of the ten textbooks. In order to ascertain the degree of accuracy of this CA estimation, the present CA results were compared with actual direct measurements of the same nine textbook corpora, quoted from Kawamura, Umamoto and Ozasa (2016). (Since all of the nine textbooks were included in the ten textbooks used in the present CA analysis, the nine textbooks were compared between the two sets, the readability sets and the CA sets except for Drill.) Table 3 and Figure 2 show the readability values of the same nine textbooks as those in the present analysis, which were measured using the then-newest readability measuring tool, Ozasa-Fukui Year Level, Ver. 3.4.2nhnc1-5 (Kawamura et al, 2016, p. 95).

Table 3. Readability Measured by OFYL

\begin{tabular}{|l|r|}
\hline Dening & 6.68 \\
\hline Union & 4.23 \\
\hline National & 3.4 \\
\hline Globe & 3.22 \\
\hline StandardT & 3.11 \\
\hline StandardP & 3.1 \\
\hline Seisoku & 3.07 \\
\hline J \& B & 1.51 \\
\hline Sunshine & 1.49 \\
\hline
\end{tabular}




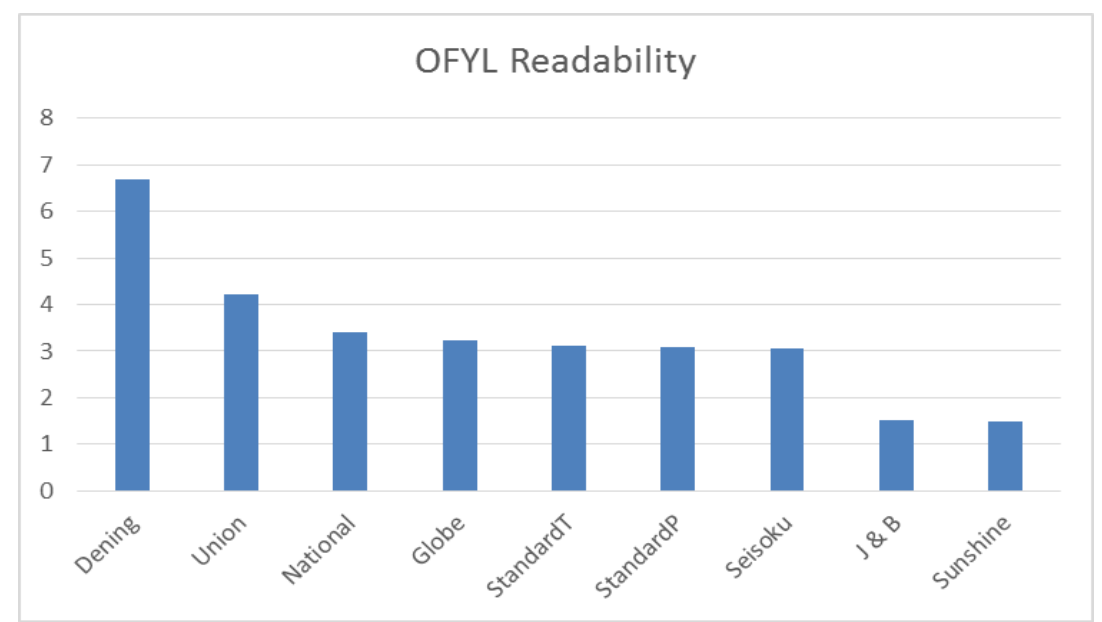

Figure 2. Readability Measured by OFYL

As it is clear in Tables 2 and 3, and Figures 1 and 2, when the two sets were compared in terms of their decreasing order, they corresponded to each other almost perfectly except for Globe and StandardT. It could be stated that the indirect readability estimation of the CA is fairly accurate, almost as accurate as the estimation of the direct measuring tool, on two grounds. First, the accuracy (prediction rate, $\mathrm{r}^{\wedge} 2$ ) of Ozasa-Fukui Year Level, Ver. 3.4.2nhnc1-5 is 0.8802 or $88.02 \%$, which means that there are about $12 \%$ chances of errors in its estimation itself (Kawamura et al, 2016, p.88). Second, the nine corpora used in the two analyses were not the same in the strict sense of the word; in the present $\mathrm{CA}$, the whole corpora were used without any kinds of modification while in the direct readability measurement, the corpora were "cleaned" or processed, in which unnecessary parts of the passages were deleted so that only "normal" sentences could be measured. These facts considered, it must be concluded that the estimation of the present CA judgment of the ten corpora is surprisingly accurate, accurate enough for the purpose of the present analysis.

This conclusion, or the judgment of the Dim 1 results could be generalized to the other four dimensions (Dims 2 to 5); although they are indirect estimation of the textbook categories, it could be reasonably accurate and trustworthy estimation and interpretation of their features.

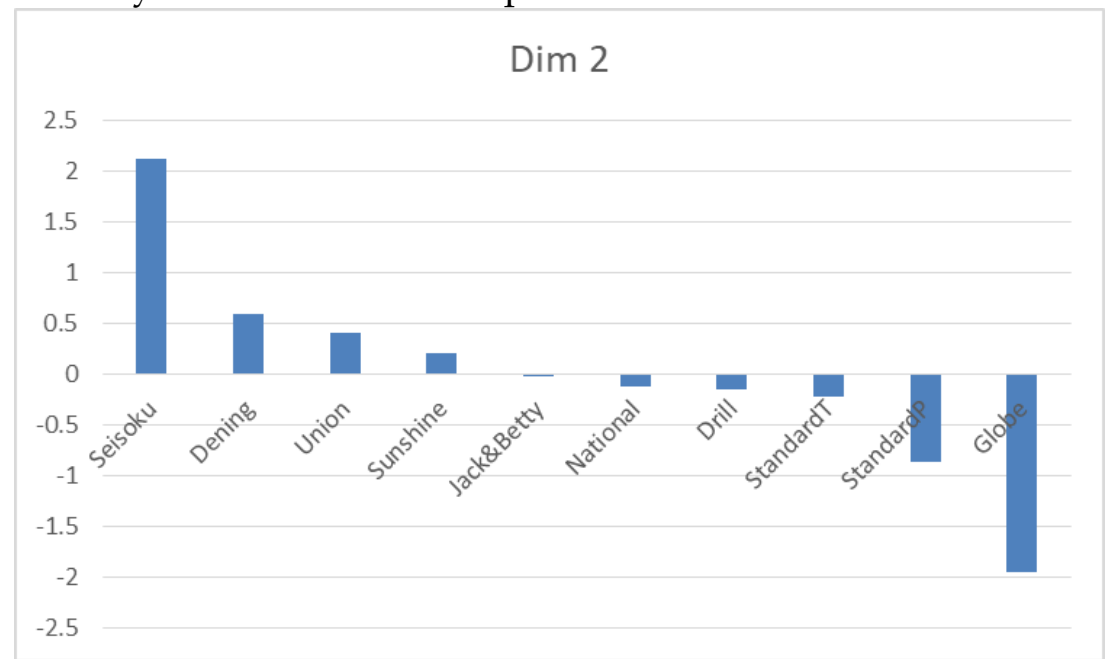

Figure 3. Values of Ten Textbooks on Dim 2 
On the second dimension, as it is also clear in Table 2 and Figure 3, the value is the highest for Seisoku (2.129), and the lowest for Globe (-1.953) and the second lowest for StandardP (-0.862), the other seven coming in between them. In decreasing order, the ten textbooks were: Seisoku $>$ Dening $>$ Union $>$ Sunshine $>$ Jack $\mathcal{E}$ Betty $>$ National $>$ Drill $>$ StandardT $>$ StandardP $>$ Globe. The difference of the values among the ten textbooks could best be explained by the category of 'drill-centered vs. natural structure'; in Seisoku, linguistic items were organized so that a small number of grammatical and lexical items were selected, graded and carefully embedded into the text / discourse for each lesson or unit while in Globe this kind of linguistic control is rather loosely observed, giving more freedom for natural communication and communicative activity. In other words, Seisoku observes the principle of a step-by-step progression, while Globe observes the principle of freer communication as a basic strategy of EFL textbook organization. For this reason, the second dimension was termed 'drillcentered vs. natural structure.' The contribution rate of the second dimension was $16.8 \%$.

Also, on the third dimension, as it is also clear in Table 2 and Figure 4, the value is the highest for Sunshine (1.628), the second highest for National (1.546), and the lowest for Seisoku (-1.413), the second lowest for Globe (-1.181), the other seven coming in between them. In decreasing order, the ten textbooks were: Sunshine $>$ National $>$ Union $>$ StandardT $>$ Jack \& Betty $>$ Dening $>$ Drill $>$ StandardP $>$ Globe $>$ Seisoku. The differences / similarities among these ten textbooks could best be explained by the category of 'multi-logue vs. monologue'. In Seisoku (the lowest) and Globe (the second lowest), almost all of the texts take the form of 'my monologue' or 'my depiction,' in which the subject 'I' alone talk or depict; in contrast, in Sunshine (the highest) and National (the second highest), almost all of the texts take the form of intercommunication among two or more interlocutors. In contrast, Drill and Jack \& Betty, and others, whose values lie in between the two contrasting ends, are comprised of various kinds of styles such as monologue, dialogue, passages, structure-based pattern practices, etc. For this reason, this dimension was termed 'multi-logue vs. monologue' discourse. The contribution rate of the fourth dimension was 0.138 , covering only the $13.8 \%$ of the whole contribution.

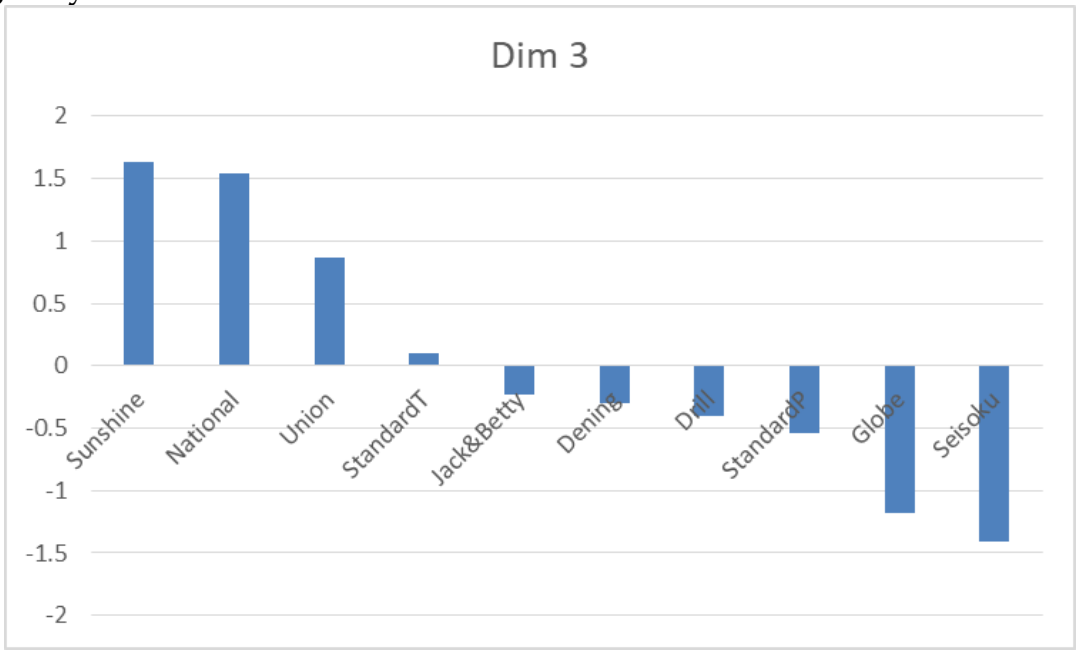

Figure 4. Values of Ten Textbooks on Dim 3 
On the fourth dimension, as it is also clear in Table 2 and Figure 5, the value is the highest for Dening (2.039) and the second highest for Sunshine (1.019) , while it is the lowest for National (-1.788). In decreasing order, the ten textbooks were: Dening $>$ Sunshine $>$ StandardP $>$ Jack $\mathcal{E}$ Betty $>$ StandardT $>$ Union $>$ Drill $>$ Globe $>$ Seisoku $>$ National. The differences / similarities among these ten textbooks could best be explained by the category of 'redundant vs. concise texts.' In Dening (the highest) and Sunshine (the second highest), for example, a considerably large part of the text are long and tends to sound redundant although sentences are syntagmatic and cohesive, while in National (the highest), the dialogues and expository passages are generally brief and comprehensive without tedious repetitive drill-like sentences. For this reason, the fourth dimension was termed 'redundant vs. concise texts.'

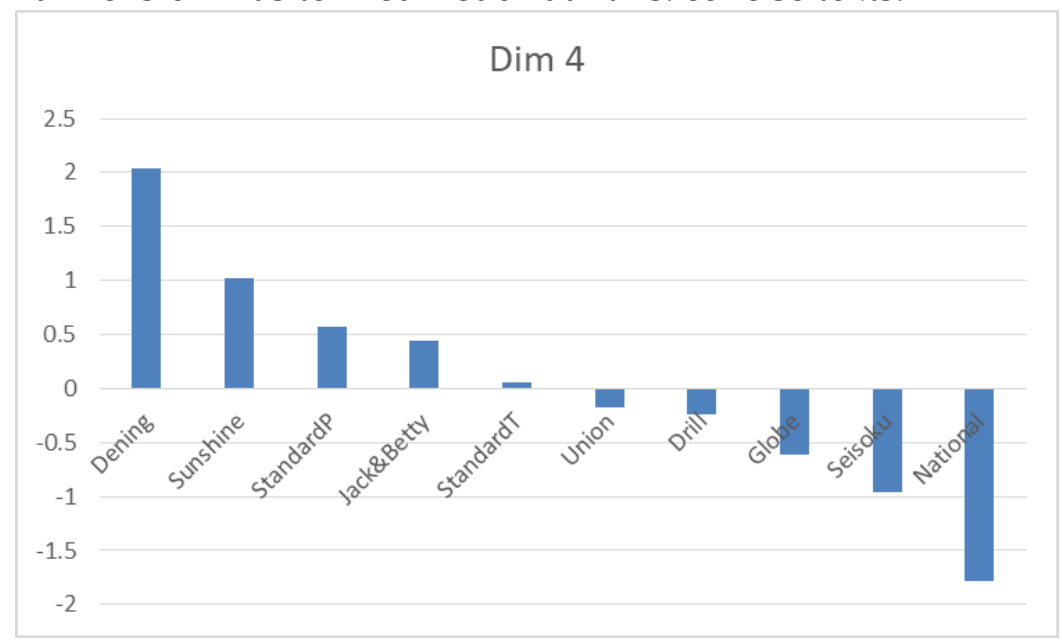

Figure 5. Values of Ten Textbooks on Dim 4

On the fifth dimension, as it is also clear in Table 2 and Figure 6, the value is the highest for Globe (1.441) and the second highest for Sunshine (1.159), while it is the lowest for Drill (-2.044), and the remaining seven textbooks are coming in between them. In decreasing order, the seven textbooks were: Globe $>$ Sunshine > Seisoku > Dening > Jack \& Betty > National > StandardT > Union > StandardP $>$ Drill. The differences / similarities among these ten textbooks could best be explained by the category of 'children's vs. adults' viewpoint.' In Globe (the highest) and Sunshine (the second lowest), a considerably large part of the texts are motivated and predominated by the viewpoints of young children or learners, while in Drill (the lowest), for example, the dialogues and expository passages are generally motivated and predominated by adults' or instructors' viewpoints. For this reason, the fifth dimension was termed 'children's vs. adults' viewpoint.' 


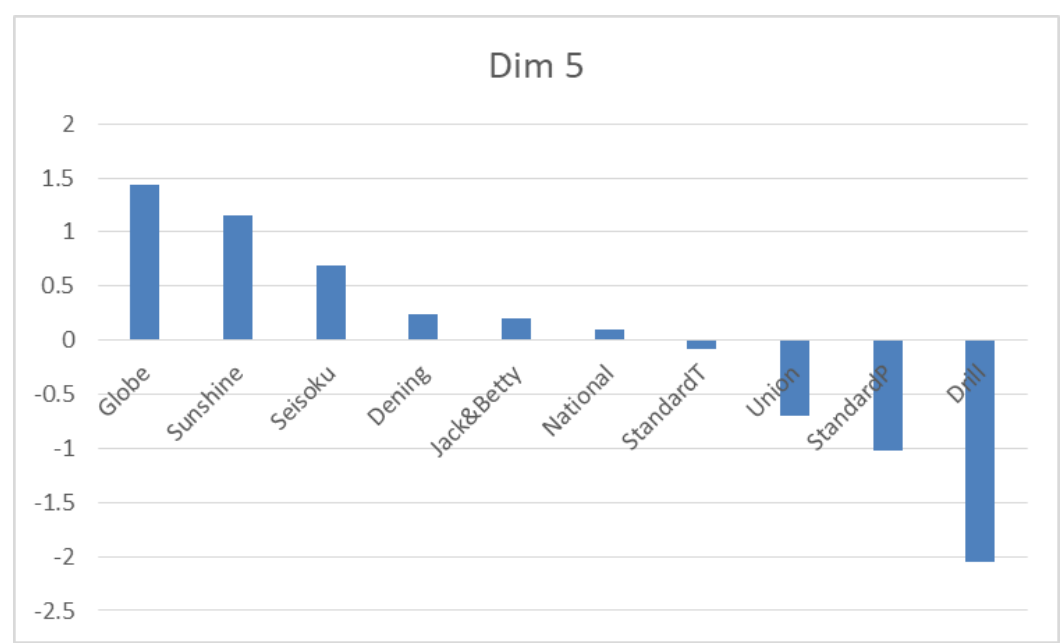

Figure 6. Values of Ten Textbooks on Dim 5

As a summary of the above descriptions and discussions, Figure 7 visualizes the spatial relationships among the ten nominal variants or textbooks in focus, in which the $x$-axis represents the first dimension or the axis of 'difficult vs. easy texts' and the $y$-axis the second dimension or the axis of 'drill-centered vs. natural structure.' As it is clear in Figure 7, the ten textbooks can be classified into four category groups, i.e., Group 1 ( + on $x$-axis, around 0 on $y$ axis), Group 2 (- on $x$-axis, around 0 on $y$-axis), Group3 (around 0 on $x$-axis, - on $y$-axis) and Group 4 (around 0 on $x$-axis, + on $y$-axis). In Group 1 (difficult text and neutral in terms of 'drill-centered vs. natural structure'), there clustered were four textbooks, i.e., Dening, Union, National and StandardT. In Group 2 (easy text and neutral in terms of 'drill-centered vs. natural structure'), there clustered were three textbooks, i.e., Sunshine, Jack $\mathcal{E}$ Betty and Drill. In Group 3 (natural text and neutral in difficulty), there were two textbooks located, i.e., StandardP and Globe and in Group 4 (drill-centered text and neutral in difficulty), there were only one textbook located, i.e., Seisoku.

Clearly, it was only Dim 1 or the axis of 'difficult vs. easy' texts that differentiated the two big groups, Group 1 and Group 2. In other words, this large cluster consisting of the seven textbooks were divided into two groups on the basis of their locations on the difficult-easy axis. This will suggest that $\mathrm{x}$-axis (difficult vs. easy texts) can be an efficient and dominant differentiator of the historical EFL textbooks in focus. 


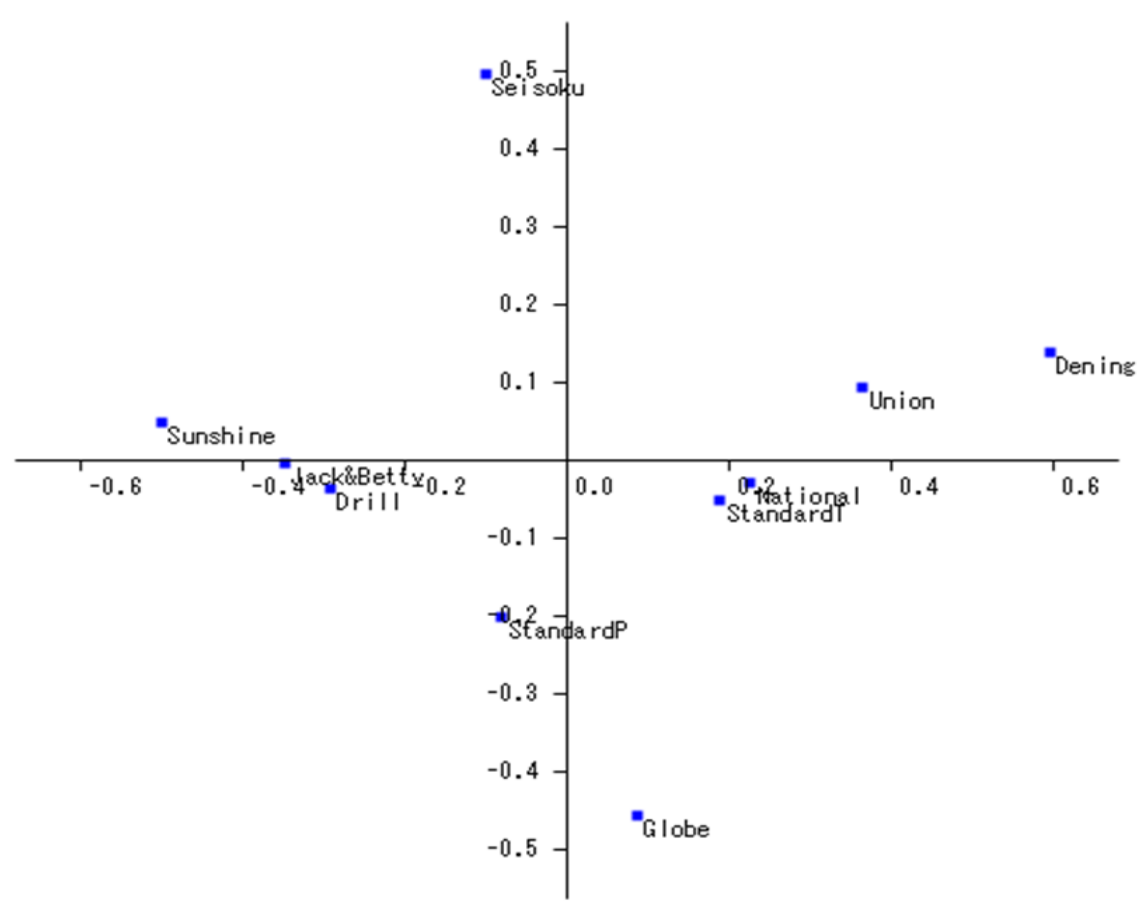

X-axis: 'difficult vs. easy' texts

Y-axis: 'drill-centered vs. natural' structure

Figure 7. CA Map of 10 Textbooks

It is true that the CA map based on the two selected major dimensions (axes) can differentiate the major features of the ten historical and current EFL textbooks but naturally it cannot differentiate all of their features detected in the analysis. In order to overcome this weakness, a new attempt was made to display the whole pictures of their features based on the values of the whole dimensions dealt with in the present analysis. It is a way of computing the actual distance (Euclidean distance) of the ten individual textbooks on each of the nine dimensions, by multiplying the weighted correlation coefficients by the coefficients proportional to the contribution rates. Using the thus-computed Euclidean distances of the ten textbooks on the nine dimensions, a cluster analysis was performed using College Analysis, by maximum distance method, so as to draw a dendrogram of the ten textbooks. Figure 8 is a dendrogram which visually summarizes the degree of similarities and differences among the ten nominal variants of the present analysis, based on the values (distances) of all of the (nine) dimensions computed in the present analysis, not based on the results of the two selected dimensions as in Figure 7.

Dendrogam is a branching diagram like biological taxonomy, representing a hierarchy of categories based on degree of similarity or the numbers of shared characteristics and graphically displays the internal cohesion and external isolation of the variants in focus, i.e., the ten textbooks in the present analysis. As it is clear in Figure 8, there identified were two groups, one (Group A) comprised of five textbooks, StandardP, Jack \& Betty, Drill, Sunshine and Seisoku, the other (Group B) comprised of five textbooks, StandardT, Union, National, Dening and Globe. In Group A, among the five constituents, Standard P 
and Jack $\mathcal{E}$ Betty are the highest in closeness or cohesion to each other at the first branch (clade), the relationship of which with Drill is the second highest at the second clade. In the same way, the lowest is the relationship of the four textbooks with Seisoku in Group A. In the same manner, in group B, the closeness between StandardT and Union is the highest and the relationship among Globe and the other four textbooks are the lowest. (It is to be noted in this respect that in dendrogram, the lower a clade is in height, the higher the relationship of the constituents in the group.)

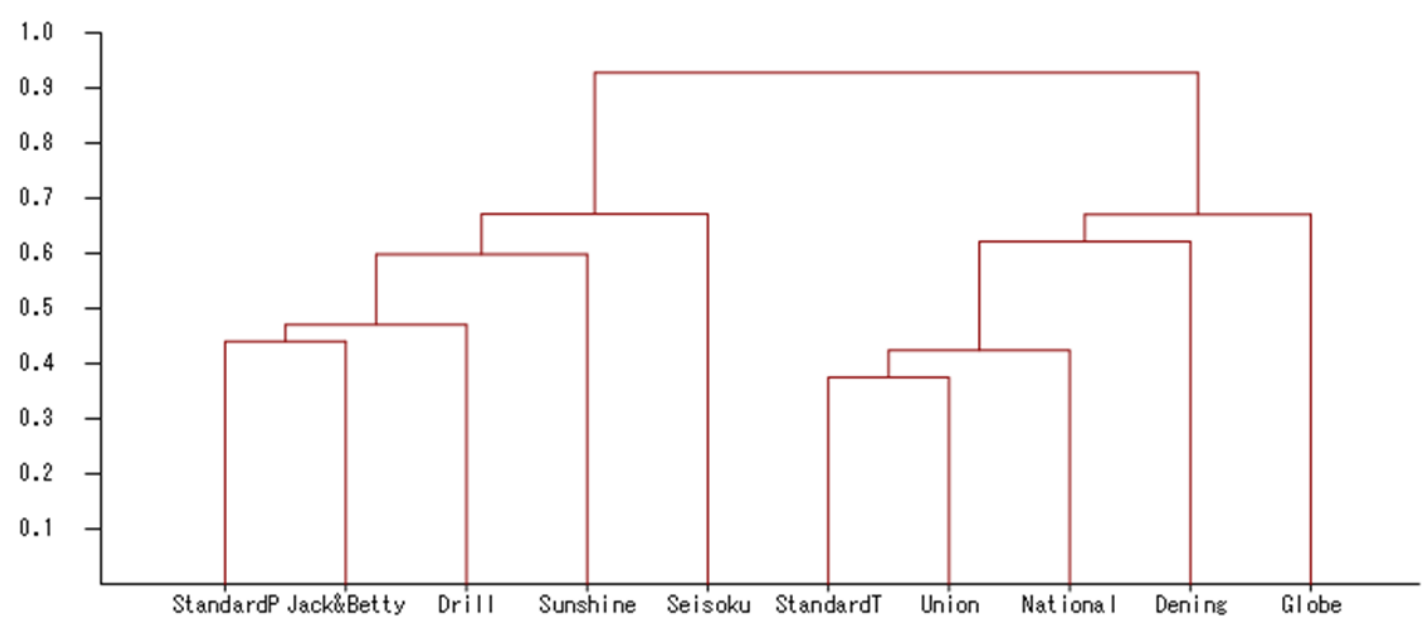

Figure 8. Dendrogram of Ten Textbooks by Maximum Distance Method

The above interpretations and discussions of the dendrogram can also be summarized using brackets, as in the following.

Group A: ((((StandardP, Jack \& Betty) Drill) Sunshine) Seisoku)

Group B: ((((StandardT, Union) National) Dening) Globe)

Interestingly, Group A consists of the textbooks authored by Japanese authors except for StandardP, while Group B consists of the textbooks authored by native-speaker authors except for StandardT and Globe.

\section{Conclusion}

So far, the results of the CA has been analyzed, interpreted and discussed, focusing on the characteristic features, similarities and differences among the nine textbooks with their current counterpart, which proved that CA is powerful enough to quantitatively analyze their features. As a summary of the results and discussions of the present CA, the RQs were answered as in the following.

RQ 1. How similar / different in contents are the ten Japanese EFL textbooks to/from each other? The CA results proved capable of differentiating the features of the ten textbooks quantitatively, specifying their similarities and differences. In particular, the CA map comprised of the two major dimensions indicated that the ten textbooks can be divided into four groups and that it was only Dimension 1 or the axis of difficult vs. easy texts that differentiated the two major groups. The dendrogram, which was comprised of the nine dimensions (axes), indicated that the ten textbooks could be divided into two groups, Group 
A and Group B based on the mutual similarities / differences (distances) among the ten textbooks.

RQ 2. How similar / different in contents are the nine Japanese historical EFL textbooks to/from their current counterparts? When compared with their current counterpart, Sunshine, all the textbooks, except for Seisoku, StandardP and Globe, proved to be similar to each other in terms of the 'drillcentered vs. natural' category (Dim 2). This suggests that the six historical textbooks are similar to their current counterpart, Sunshine and that the other three textbooks are different from their current counterpart in terms of the 'drillcentered vs. natural' category.

RQ 3. What kinds of dimensions explain the similarities / differences among the ten textbooks? The explaining categories (dimensions, axes) proved to be the 'difficult vs. easy' axis (Dim 1), the 'drill-centered vs. natural' axis (Dim 2), 'multi-viewpoints vs. single viewpoints' axis (Dim 3), 'redundant vs. concise' axis (Dim 4) and 'children's vs. adults' viewpoint' axis (Dim 5).

Finally, it was concluded that the CA proved to be powerful enough to quantitatively analyze, interpret and clarify the characteristic features, similarities and differences among the nine historical textbooks and their current counterpart.

\section{References}

Hosaka, Y., Abe, N., Uenishi, K. \& Ozasa, T. (2008). To-infinitive in Japan's EFL textbooks: A diachronic comparison. Proceedings of $4^{\text {th }}$ International ICTATLL (ICT in the Analysis, Teaching and Learning of Languages) Workshop, University of Colombo, Sri Lanka, September, 2008, 1-5.

Hosaka, Y., Erikawa, H., Miura, S. \& Ranjan, C. K. (2008). Verbals in Japan's first-year EFL textbooks: A diachronic quantitative comparison. In Weir, G., \& Ozasa, T., (Eds.), Studies in Language and Textbook Analysis. (pp. 143-148) Glasgow: University of Strathclyde Publishing.

Kawamura, K.., Umamoto, T., Matsuoka, H. \& Ozasa, T. (2017). Meiji-Showaki no eigo kyokasho no ri-dabiriti bunseki: Jogakko kyokasho o chushin tosite [Readability of four English textbooks used from the Meiji to Showa Era in Japan: Focusing on girls' middle school textbooks]. Journal of ICTATLL (ICT in the Analysis, Teaching and Learning of Languages) Japan, 4, 71 - 85.

Kawamura, K., Umamoto, T. \& Ozasa, T. (2016). Meijiki-gendai no eigo kyokasho 9shu no ri-dabiriti bunseki: Ozasa-Fukui Year Level, Ver. 3.4.2nhnc1-5 niyoru bunseki [Readability of nine English textbooks used from the Meiji Era to the present day in Japan: An analysis with the use of Ozasa-Fukui Year Level, Ver. 3.4.2nhnc1-5]. Journal of ICTATLL (ICT in the Analysis, Teaching and Learning of Languages) Japan, 3, 85 - 99.

Matsuoka, H., Umamoto, T., Ozasa, T. \& Motooka, N. (2008). A diachronic quantitative analysis of the first-year EFL textbooks of Japan: With a focus on the introduction of verbs. In Weir, G., \& Ozasa, T., (Eds.), Studies in Language and Textbook Analysis. (pp. 29-36). Glasgow: University of Strathclyde Publishing.

Nakamura, T \& Ozasa, T. (2007). Diachronic and synchronic analysis of English textbooks: With a focus on the gender awareness. Proceedings of the 12th Conference of Pan-Pacific Association of Applied Linguistics, Pataya, Thailand, 19-21, December, 2007, 126-129.

Ozasa, T. \& Erikawa, H. (Eds.) (2004). Eigo Kyokasho no Rekishiteki Kenkyu [A Historical Study of [Japanese] English Textbooks]. Tokyo: Jiyusha. 
Ozasa, T., Fukui, M. \& Watanabe, K. (2015). Development of a readability index attuned to the new English course of study of Japan (3): Development of Ozasa-Fukui Year Level, Ver. 3.4.2nhnc1-5. Pixel ed. Conference Proceedings, $8^{\text {th }}$ International Conference of ICT for Language Learning, Florence, Italy, 12-13 November, 2015, 349354.

Ozasa, T. \& Nakamura, Y. (2001). Meiji, Taisho, Showa Shoki no Eigo Kyokasho nikansuru Kenkyu: Shitsuteki Bunseki to Kaidai [A Study of [Japanese] Historical English Textbooks Published in the Meiji, Taisho and Showa Era: A Qualitative Analysis and Explanatory Notes]. Hiroshima: Keisuisha.

Ozasa, T., Umamoto, T., Matsuoka, H., \& Motooka, N. (2008). Vocabulary of the firstyear EFL textbooks of Japan: A diachronic quantitative comparison. In Weir, G., \& Ozasa, T., (Eds.), Studies in Language and Textbook Analysis. (pp. 149-155). Glasgow: University of Strathclyde Publishing.

Ozasa, T., Watanabe, K. \& Fukui, M. (2016). Development of a readability index attuned to the new English course of study of Japan (4): Development of Ozasa-Fukui Year Level, Ver. 3.5nhnc1-6. Conference Proceedings, ICSSB (International Conference on Social Sciences and Business), Tokyo, Japan, August 25 - 27, 2016, 385394.

Sakamoto, M., Watanabe, K. \& Ozasa, T. (2017). A Correspondence analysis of five Japanese historical English-as-a-foreign-language textbooks. Conference Proceedings of ICSSB (International Conference on Social Science and Business), Okinawa, Japan, July 25 - 27, 2017, 132-144.

Tono, Y. (2000). A Corpus-based analysis of interlanguage development: Analysing partof-speech tag sequences of EFL learner corpora. In Lewandowska-Tomaszczyk, B \& Melia, P. J. (Eds.), Proceedings of PALC' 99: Practical Applications in Language Corpora, University of Lodz, Poland, 15-18 April, 1999, 323-340.

Uenishi, K., Watanabe, K. \& Ozasa, T. (2017). Correspondence analysis of three Japanese EFL textbooks: In reference to the improvement of a criterion measure for a readability tool. Conference Proceedings, ICEPL (International Conference on Education, Psychology, and Learning), Sapporo, Japan, August 23-25, 2017, 49-60.

Watanabe, K., Asai, T. \& Ozasa, T. (2017). A correspondence analysis of five Japanese historical English-as-a-foreign-language textbooks. Conference Proceedings, ICEPL (International Conference on Education, Psychology, and Learning), Sapporo, Japan, August 23-25, 2017, 61-73.

Weir, G. \& Ozasa, T. (2007). Estimating naturalness in Japanese English textbooks. Proceedings of the 12th Conference of Pan-Pacific Association of Applied Linguistics, Pattaya, Thailand, 19-21, December, 2007, 130-133.

Weir, G. \& Ozasa, T. (Eds.) (2007). Texts, Textbooks and Readability. Glasgow: University of Strathclyde Publishing.

Weir, G. \& Ozasa, T (2008). Multiword vocabulary in Japanese ESL texts. Proceedings of the 13th Conference of Pan-Pacific Association of Applied Linguistics, University of Hawaii, U.S.A., 20-22 August, 2008, 63-66.

Weir, G. \& Ozasa, T. (Eds.) (2008). Studies in Language and Textbooks Analysis. Glasgow: University of Strathclyde Publishing.

Weir, G. \& Ozasa, T. (2009). Verb form usage in Japanese EFL texts. Proceedings of the 14th Conference of Pan-Pacific Association of Applied Linguistics, Kyoto, Japan, 31 July - 2nd August, 2009, 101-104.

Weir, G. \& Ozasa, T. (2010). Learning from analysis of Japanese EFL texts. Educational Perspectives (Journal of the College of Education, University of Hawaii at Manoa), 43, 1 \& 2, 56-66. 